\title{
MECHANICAL PROPERTIES OF A STRUCTURAL POLYURETHANE FOAM AND THE EFFECT OF PARTICULATE LOADING
}

\author{
S.H. GOODS*, C.L. NEUSCHWANGER* and L.L. WHINNERY*
}

*Sandia National Laboratories, Livermore, CA, 94550
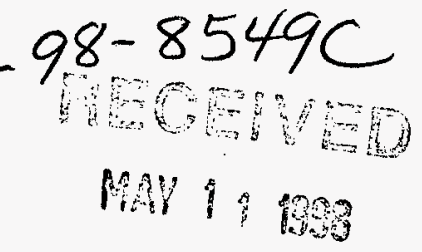

\section{ABSTRACT}

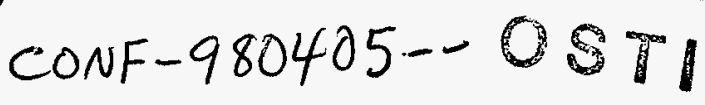

The room temperature mechanical properties of a closed-cell, polyurethane encapsulant foam have been measured as a function of foam density. Tests were performed on both unfilled and filler reinforced specimens. Over the range of densities examined, the modulus of the unloaded foam could be described by a power-law relationship with respect to density. This power-law relationship could be explained in terms of the elastic compliance of the cellular structure of the foam using a simple geometric model found in the literature. The collapse stress of the foam was also found to exhibit a power-law relationship with respect to density. Additions of an aluminum powder filler increased the modulus relative to the unfilled foam.

\section{INTRODUCTION}

Polyurethane foams are used as encapsulants to provide environmental isolation and to mitigate harsh thermal and mechanical shock environments. For the latter application it is necessary to fully characterize the tension and compression properties of the chosen foam system. Historically, a principal constituent of many polyurethane foam systems has been toluene diisocyanate (TDI), a suspect human carcinogen. It is desirable, therefore, to replace such potentially hazardous constituents. To that end, the CRETE foam system (1), which uses non-TDI components was developed and is the subject of the work reported here. The purpose of the current work was three-fold. Our first goal was to measure "quasi-static" tensile and compressive mechanical properties of CRETE, specifically, elastic modulus and compressive collapse stress. A second goal was to relate certain of these properties to the microstructure and density of the foam through simple microstructural models based on cell strut flexure and buckling. Lastly, the effect of a metal powder additive on the modulus of foam was measured and compared to the unloaded foam formulation.

\section{EXPERIMENTAL}

\section{Formulation of CRETE Foam System}

CRETE is a rigid, closed cell, water blown polyurethane foam. As with other polyurethane foams, reaction of the water with isocyanate produces carbon dioxide that expands the foam so that no physical blowing agents are needed. The density of CRETE foam is varied by changing the amount of added water, thereby controlling the amount of rise in the foam (through the evolution of $\mathrm{CO}_{2}$ ). The amount of catalyst and surfactant can also be varied slightly to optimize processing and foam properties.

CRETE is formulated from the following constituents:

- Voranol 490:

- Isonate 143L:

A polyether polyol

- DC193:

A modified methylene diisocyanate (MMDI)

- Polycat 17:

A silicone glycol copolymer surfactant.

- Distilled water:

A tertiary amine catalyst.

Added as a chemical blowing agent producing carbon dioxide.

Foam was also processed with additions of a gas-atomized aluminum powder. The powder particles were spherical in shape and had mean diameters in the $2-4 \mu \mathrm{m}$ range. The powder was added to the polyol and diisocyanate constituents of the foam and then all of the components were mixed together in the usual fashion. SEM revealed that the powder particles were well incorporated into the cell wall and strut structure of the foam. 


\section{DISCLAIMER}

This report was prepared as an account of work sponsored by an agency of the United States Government. Neither the United States Government nor any agency thereof, nor any of their employees, makes any warranty, express or implied, or assumes any legal liability or responsibility for the accuracy, completeness, or usefulness of any information, apparatus, product, or process disclosed, or represents that its use would not infringe privately owned rights. Reference herein to any specific commercial product, process, or service by trade name, trademark, manufacturer, or otherwise does not necessarily constitute or imply its endorsement, recommendation, or favoring by the United States Government or any agency thereof. The views and opinions of authors expressed herein do not necessarily state or reflect those of the United States Government or any agency thereof. 


\section{Mechanical Testing}

Room temperature uniaxial tension and compression mechanical properties were evaluated as a function of foam density. Right circular cylinders, $50.8 \mathrm{~mm}$ long and $28.7 \mathrm{~mm}$ in diameter, were cored from $20 \mathrm{~cm}$ diameter pours of the foam with the cylinder axis parallel to the direction of the foam rise. None of the cored samples included the foam skin or any material from within 3 mm of the surface. These simple right circular cylinders served as the compression specimens. For tension testing, the cylinders were bonded to pull studs. Gauge sections measuring $25.4 \mathrm{~mm}$ long and $19.0 \mathrm{~mm}$ in diameter were then machined into the foam cylinders. All specimens were instrumented with a mechanically attached extensometer for displacement measurement. The reduced gauge section in the tension specimens insured that failure occurred within the specimen between the attachment points of the extensometer and not at the bondline between the foam and the pull studs. Tests were conducted on specimens having densities between $0.1 \mathrm{~g} / \mathrm{cm}^{3}$ and approximately $0.8 \mathrm{~g} / \mathrm{cm}^{3}$ at an initial strain rate of $1.7 \times 10^{-4} \mathrm{~s}^{-1}$.

Modulus, $E^{\star}$, as a function of foam density was determined for specimens tested in both tension and compression. An additional parameter for compression testing, the collapse stress, $\sigma_{\mathrm{C}}{ }^{*}$, a broad plateau region subsequent to linear loading, was also determined as a function of density.

\section{RESULTS AND DISCUSSION}

\section{Modulus and Strength of Foam}

The modulus as a function of foam density is shown in Figure 1 for both tension and compression testing. Mechanical properties during the early stages of compression (up to the onset of collapse) are essentially identical to those observed in tension. The modulus of the foam exhibits a power-law dependence with respect to foam density as:

$$
E^{*} \propto(\rho *)^{n}
$$

where $E^{*}$ is the modulus of the foam, $\rho^{*}$ is the foam density and $n$ is the density exponent. Over the range of density shown in Figure 1, the data are well fit for a density exponent of $n=1.6$.

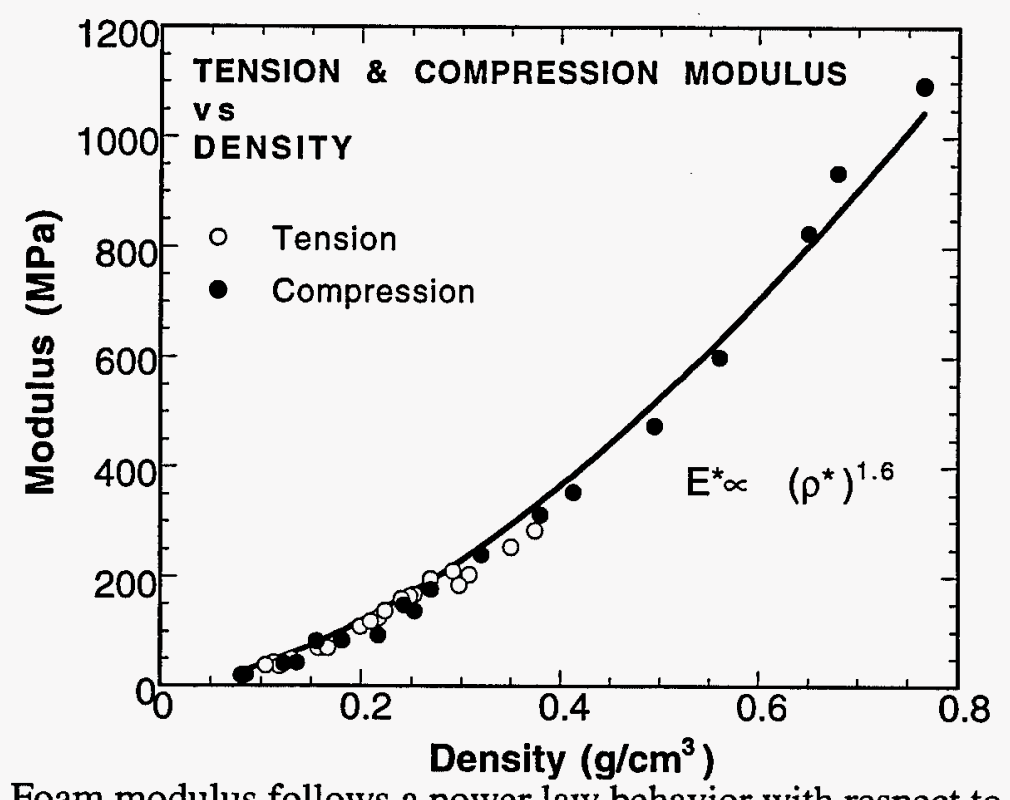

Figure 1. Foam modulus follows a power law behavior with respect to density.

Foam specimens tested in compression show a relatively abrupt change in the initial slope of the loading curve followed by a sustained plateau region. At the lower densities (up to approximately $0.3 \mathrm{~g} / \mathrm{cm}^{3}$ ), the stress after the plateau actually drops slightly, giving rise to a yield 
point-like behavior. The inset in Figure 2 is typical of this behavior. The broad plateau region results from the collapse or cell wall buckling of the foam (2). The stress begins to increase subsequent to this plateau region as the foam begins to densify. Unlike the tests performed in tension, fracture is inhibited by the absence of tensile stresses and as a result, engineering strains in excess of $90 \%$ have been measured with little observable indication of fracture. Figure 2 shows the plateau stress for the foam specimens as a function of density. This plateau stress, also called the collapse stress, $\sigma_{\mathrm{C}}{ }^{*}$, is important in the design of cushions for shock or impact mitigation as it represents the onset of the mechanical instability of the foam microstructure (2). It too, exhibits a power-law dependence with respect to foam density.

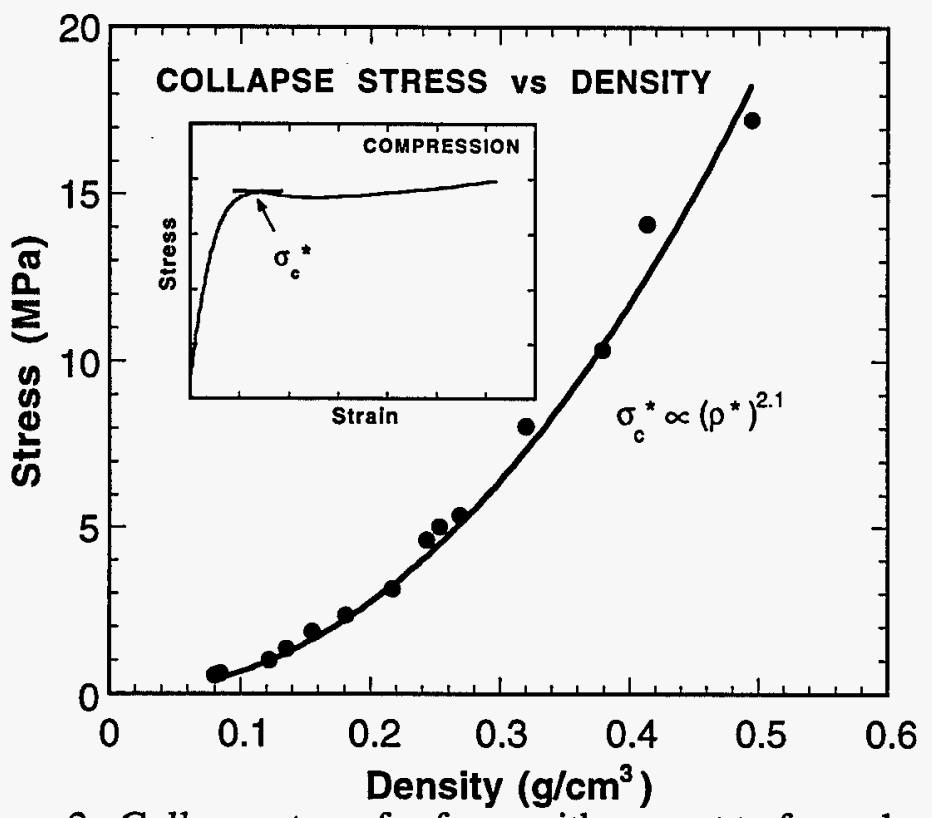

Figure 2. Collapse stress for foam with respect to foam density.

\section{Prediction of Modulus and Strength}

The dependence of both the modulus and the collapse stress of a cellular foam on density can be understood in terms of the mechanical properties of the polymer material from which the cell struts (and for a closed-cell foam, the cell walls) are made and the deformation mechanics of the cellular structure itself. Elastic moduli are related principally to the bending stiffness of the members comprising the cellular structure while the collapse is caused by the buckling of these same members.

The important cell strut/wall properties are the solid polymer density, $\rho_{s}$, and its modulus, $E_{s}$. The important structural features for the analysis of the modulus and the collapse stress are the relative density of the foam, $\rho^{*} / \rho_{s}$, (as before, $\rho^{*}$ is the density of the foam) and whether or not the cells are open or closed. In this regard a parameter, $\phi$, is defined as the fraction of material in the cell struts (2). For an open cell foam, $\phi=1$, while for a closed cell foam, where some of the polymer is in the cell walls, it is less than 1.

The principal mechanism of linear-elastic deformation for foams was first identified correctly by Menges and Knipschild as cell wall bending (3). They also pointed out that open and closed cell foams have similar stiffness because it is the cell wall edges or struts, rather than the thin cell wall membranes, that carry the majority of an imposed load. Ko also identified cell wall bending as the controlling factor in the determination of the modulus but the model presented in that work was complicated by the extremely complex cell geometry (4). Similarly, Patel and Finnie report in great detail the geometrical requirements necessary for various three dimensional structures to fill space (5). They describe how no regular polyhedra can meet all of the requirements for mutually shared edges while satisfying compatibility. The analysis of the mechanics of the cell structures based upon rigorous compatibility leads to intractable mathematics. 
A much simplified model of an open cell foam has been put forth by Gibson and Ashby $(2,6)$ in which the foam is modeled as an array of cubic cells which are staggered so that corners of one cell rest upon the midpoint of adjacent cells. Such a structure corresponds neither to the actual geometric characteristics of a real foam nor can it be reproduced to fill space. This "unit cell" does, however, capture the critical physical processes that govern the deformation and structural stability of a cellular structure. In the original analysis, the elastic modulus is found to be governed by the flexure of cell struts normal to an applied load and follows the relationship:

$$
\frac{E^{*}}{E_{S}} \approx \phi^{2}\left(\frac{\rho^{*}}{\rho_{S}}\right)^{2}+(1-\phi) \frac{\rho^{*}}{\rho_{S}}
$$

The quadratic term describes the contribution of the cell struts to the modulus and is the same for open cell foams. The linear term accounts for the cell walls. Note that an additional term accounting for internal (atmospheric) gas pressure within the cells is ignored in Equation 2.

The data shown in Figure 1 can be directly compared to Equation 2 using known values for $\rho_{s}, E_{s}$ and $\phi$. For the density of the solid we use a value of $1.2 \mathrm{~g} / \mathrm{cm}^{3}(7)$. The value for the

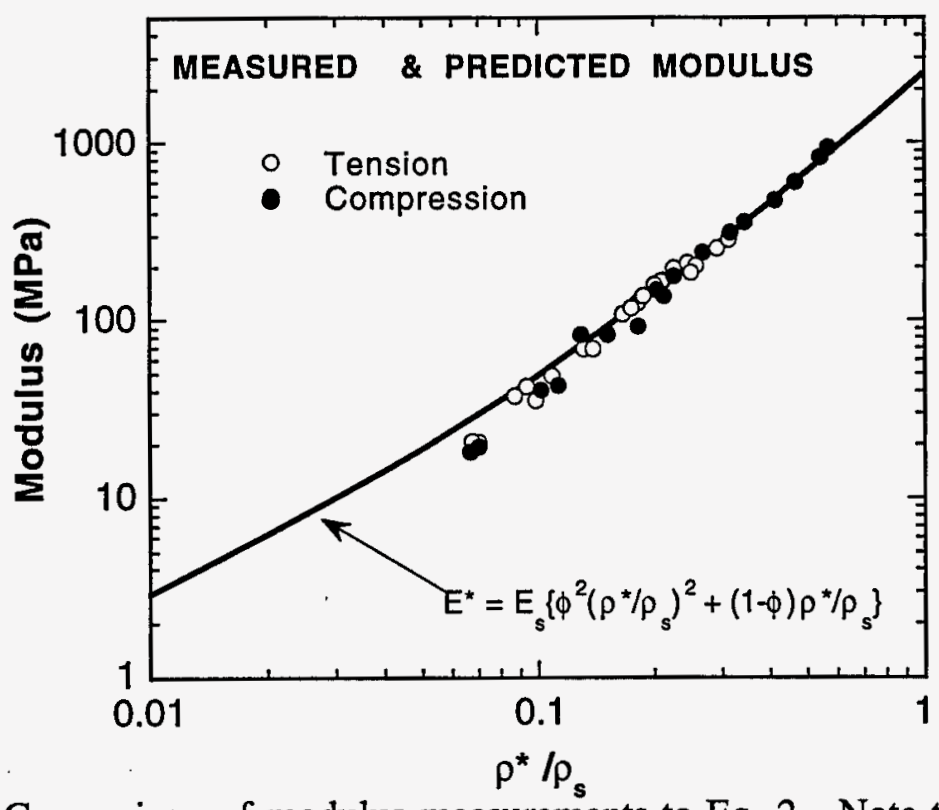

Figure 3. Comparison of modulus measurements to Eq. 2. Note that density is normalized by the density of the solid polymer.

modulus of solid polyurethane is less well established and varies considerably depending on the precise formulation, processing conditions and product form. We use a value within the range of those reported, $2.7 \mathrm{GPa}(6,7,8)$. The last term, $\phi$, has been measured for polyurethane foams and found to equal approximately 0.9 (9). Note that in keeping with the form of Eq. 2, the abscissa is now the normalized density, $\rho^{*} / \rho_{\mathrm{s}}$. It is clear from Figure 3 that the model well represents the data over the range of densities examined experimentally, predicting both the absolute values of the modulus as well as its density dependence.

The dependence of the collapse stress on foam density can be examined in a similar fashion. When an axial load is small, the compressed columns that comprise the cell struts parallel to the applied load are laterally stable. However, as the load is increased, these columns become unstable and lateral displacements tend to remain. This instability is termed "lateral buckling" and the applied load necessary to cause it is called the "Euler buckling load". The derivation of the Euler buckling load is a well known problem in mechanics (10) and its application to a foam structure yields a prediction of collapse as $(2,6)$ : 


$$
\frac{\sigma_{c}^{*}}{E_{s}}=C\left(\frac{\rho^{*}}{\rho_{s}}\right)^{2}\left(1+\left(\frac{\rho^{*}}{\rho_{s}}\right)^{1 / 2}\right)^{2}
$$

The constant $\mathrm{C}$ contains all of the geometric constants and from a comprehensive analysis of the data in the literature, Gibson and Ashby $(2,6)$ have determined that $C=0.03$. Using this value and the same values for $\rho_{s}$ and $E_{s}$ as used before, we compare the collapse stress measurements in Figure 2 to Equation 3 . The results are shown in Figure 4. Notwithstanding the uncertainty in some of the parameters, the agreement between the measured and predicted collapse stress is quite good. As with the modulus, Equation 3 predicts the density dependence of $\sigma^{*}{ }_{c}$ quite well. In this instance, though, the absolute values of the collapse stress are somewhat overestimated by Equation 3.

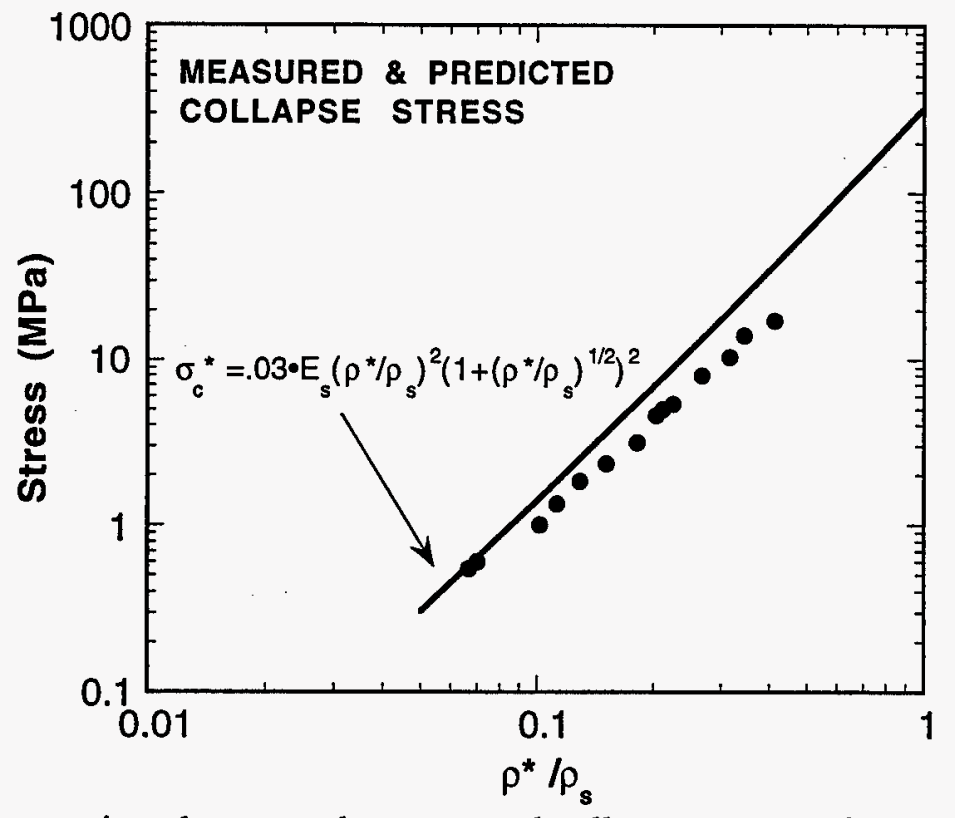

Figure 4. Comparison between the measured collapse stress and Equation 3.

\section{Effect of Particle Loading on Modulus}

The influence of a rigid filler phase on the modulus of the foam is shown in Figure 5. This Figure compares the original modulus data for CRETE shown in Figure 1 to specimens containing controlled additions of the aluminum powder filler. Note that the original CRETE data is presented as the bold solid line representing the best fit through the data and that the individual data points have been omitted for clarity. The measured moduli for the specimens containing $10 \mathrm{wt} . \%, 30$ wt. $\%$ and $50 \mathrm{wt} . \%$ are also shown in this Figure. The term, $\rho^{*} / \rho_{\mathrm{s}}$ continues to refer to the ratio of the density of the polymer within each specimen to that of solid polyurethane. In this way we can directly compare the effects of $\mathrm{Al}$ additions on the modulus at constant relative polymer density.

Because the aluminum powder is well dispersed within the polymer, the foam struts and walls should act as particle-reinforced structural elements. The modulus of such "composite" structures typically depends on the volume fraction of the additive and its modulus relative to that of the host matrix $(11,12)$. Since the modulus of aluminum is approximately 30 -times greater than that of the polyurethane in which it is dispersed, we expect, that additions of $\mathrm{Al}$ powder would yield a foam with an increased overall modulus.

Figure 5 shows that additions of $10 \mathrm{wt}$. \% or less of the $\mathrm{Al}$ filler have no measurable effect on the modulus of the foam. However, higher $\mathrm{Al}$ loading fractions do yield specimens with measurably increased moduli. At $30 \mathrm{wt}$.\% loading, the modulus of the filled foam is as much as twice that of the unfilled CRETE. With increasing amounts of aluminum, the modulus continues to increase relative to that of the unmodified foam. At $50 \mathrm{wt}$. \% loading, a three-fold increase in modulus can be realized. 


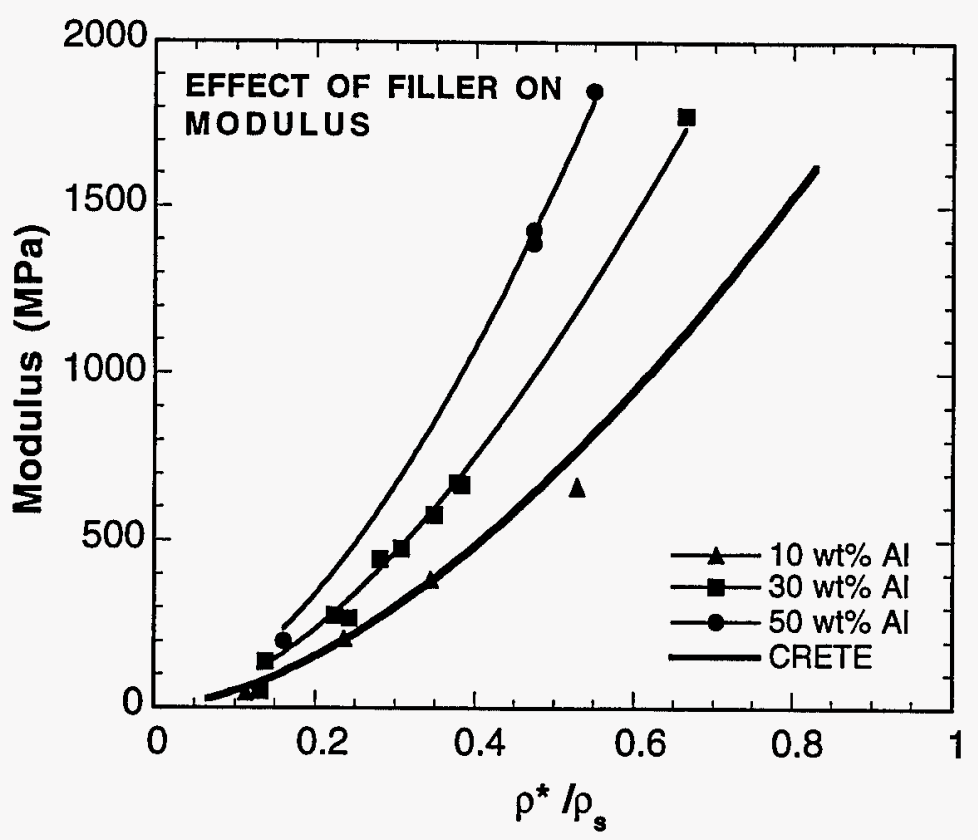

Figure 5. Effect of aluminum powder on modulus of CRETE foam.

\section{CONCLUSION}

The agreement between the measured modulus and the collapse stress with those predicted by Equations 2 and 3 suggests that a model available in the literature $(2,6)$ based on a simple, idealized cell geometry, can be useful in describing certain mechanical and physical properties of foams. The agreement is remarkable in light of the simple cubic unit cell geometry chosen as the basis of the model. While not physically realistic, this cell geometry captures the density dependence of cell strut bending upon which the modulus is dependent and the cell strut buckling upon which the collapse stress of the foam is dependent. Incorporation of a metal powder into the cell structure of the foam increases the overall modulus. This increase can be rationalized in terms of the expected effect of adding a rigid particulate filler phase to a compliant matrix.

\section{ACKNOWLEDGMENT}

This work supported by DOE Contract DE-AC04-94AL85000. SEM analysis of the aluminum loaded foams was conducted by N. Y. C. Yang.

\section{REFERENCES}

1. S. H. Goods, C. L. Neuschwanger, C. Henderson and D.M. Skala, "Mechanical Properties and Energy Absorption Characteristics of a Polyurethane Foam", SAND97-8490, Sandia National Laboratories, (1997)

2. L. J. Gibson and M. F. Ashby, Proc. Roy. Soc., A382 (1982), p. 43

3. G. Menges and F. Knipschild, Polymer Engr'ing Science, 15 (1975), p. 623.

4. W. L. Ko, J. Cell Plastics, 1 (1965), p. 45.

5. M. R. Patel and I. Finnie, J. Mater., 5 (1970), p. 909.

6. L. J. Gibson and M. F. Ashby, Cellular Solids, Structure and Properties (New York, N.Y., Pergamon Press, 1988).

7. D. W. Reitz, M. A. Schuetz and L. R. Glicksman, J. Cell. Plast., 20 (1984), p. 104.

8. PLASTICS DIGEST, 10, ( D.A.T.A. Business Publishing). p. 1301-1304.

9. W. F. Roff and J. R. Scott, Fibres, Films. Plastics and Rubbers-A Handbook of Common Polymers, (London, Butterworths, 1971), p. 455.

10. W. A. Nash, Strength of Materials, 3rd Ed., Schaums Outline Series, (New York, N.Y., McGraw-Hill, Inc., 1994)

11. E. H. Kerner, Proc. Phys. Soc. (B), 69, 808 (1956).

12. T. B. Lewis and L. E. Nielson, J. Appl. Polymer Sci., 14, (1970), p.1449 
M98052861

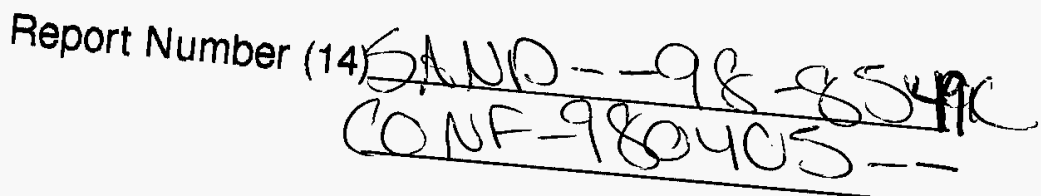

Dubl. Date (11) 199804

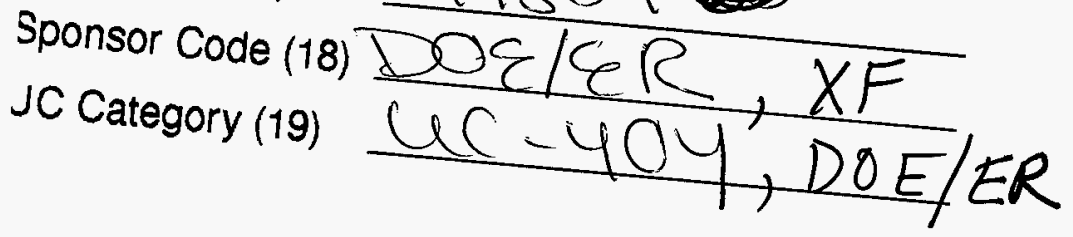

DOE 